\title{
Brief 5: Bartel Leendert van der Waerden an Grete Hermann (20.1.1934)
}

Leipzig, 20. Jan. 1934

Liebes Frl. Hermann,

Ich sprach über Ihren Brief mit Heisenberg, der meinte, er wäre einverstanden, wenn Sie hierher kämen, vorausgesetzt daß Sie nicht mehr von seiner Zeit in Anspruch nähmen als etwa 2 Stunden in der Woche. Unter ähnlichen Bedingungen stehe ich auch gerne zu Ihrer Verfügung. Wir sehen beide ein, daß eine Diskussion zwischen Ihnen und uns zur Klärung der philosophischen Fragen der Quantenmechanik für beide Teile lehrreich verlaufen könnte. Andererseits haben wir beide sehr viel anderes zu tun, und müssen unsere Zeit einteilen: daher der obige Vorschlag. - Im theoretisch-physikalischen Seminar stehen jetzt meistens nur rechnerische Probleme über Moleküle und Gitter ohne prinzipielles Interesse zur Diskussion, so daß Sie davon wenig haben würden. Philosophisch-interessierte Schüler sind, seit Weizsäcker weg ist, auch keine mehr da. Nur Heisenbergs Mittwochvorlesung (5-7) könnten Sie mit Vorteil hören: er liest jetzt über die Quantelung der Wellenfelder.

Mit den besten Grüßen

Ihr

B. Leendert Waerden

(C) Springer Fachmedien Wiesbaden GmbH, ein Teil von Springer Nature 2019

K. Herrmann (Hrsg.), Grete Henry-Hermann: Philosophie - Mathematik-

Quantenmechanik, Frauen in Philosophie und Wissenschaft. Women

Philosophers and Scientists, https://doi.org/10.1007/978-3-658-16241-2_27 